\title{
Decoupling of Early V5 Motion Processing from Visual Awareness: A Matter of Velocity as Revealed by Transcranial Magnetic Stimulation
}

\author{
Paolo A. Grasso ${ }^{1,2 *}$, Elisabetta Làdavas ${ }^{2}$, Caterina Bertini ${ }^{2}$, Serena Caltabiano², \\ Gregor Thut ${ }^{1}$, and Stephanie Morand ${ }^{1}$
}

\begin{abstract}
Motion information can reach V5/MT through two parallel routes: one conveying information at early latencies through a direct subcortical route and the other reaching V5 later via recurrent projections through V1. Here, we tested the hypothesis that input via the faster direct pathway depends on motion characteristics. To this end, we presented motion stimuli to healthy human observers at different velocities $\left(4.4^{\circ} / \mathrm{sec}\right.$ vs. $23^{\circ} / \mathrm{sec}$ ) with static stimuli as controls while applying transcranial magnetic stimulation (TMS) pulses over V5 or V1. We probed for TMS interference with objective (two-alternative forced choice $[2 \mathrm{AFC}]$ ) and subjective (awareness) measures of motion processing at six TMS delays from stimulus onset (poststimulus window covered: $\sim 27-160 \mathrm{msec}$ ). Our results for V5-TMS showed earlier interference with objective performance for
\end{abstract}

\section{INTRODUCTION}

Perception of motion is an important function of our visual system, because motion stimuli are, in many cases, also behaviorally relevant. One of the most prominent visual areas in the processing of motion signals is a relatively small portion of extrastriate visual cortex, area MT/V5. In monkeys, lesions to this area lead to severe impairments in motion direction discrimination (Newsome \& Pare, 1988; Newsome, Wurtz, Dürsteler, \& Mikami, 1985), and transcranial magnetic stimulation (TMS) of this area in humans results in transient impairments of motion processing (Sack, Kohler, Linden, Goebel, \& Muckli, 2006; d'Alfonso et al., 2002; Walsh, Ellison, Battelli, \& Cowey, 1998; Hotson, Braun, Herzberg, \& Boman, 1994; Beckers \& Hömberg, 1992).

Based on hierarchical models of visual processing in primates, V5 can be considered a relatively late area in the processing stream. After activating the retina, visual

\footnotetext{
${ }^{1}$ University of Glasgow, ${ }^{2}$ University of Bologna

*Present address: CIMeC, Centre for Mind/Brain Sciences, University of Trento, Via Delle Regole 101, 38123, Mattarello, Trentino, Italy.
}

fast motion ( $53.3 \mathrm{msec})$ than slow motion $(80 \mathrm{msec})$ stimuli. Importantly, TMS-induced decreases in objective measures of motion processing did correlate with decreases in subjective measures for slow but not fast motion stimuli. Moreover, V1TMS induced a temporally unspecific interference with visual processing as it impaired the processing of both motion and static stimuli at the same delays. These results are in accordance with fast moving stimuli reaching V5 through a different route than slow moving stimuli. The differential latencies and coupling to awareness suggest distinct involvement of a direct (i.e., colliculo-extrastriate) connection bypassing V1 depending on stimulus velocity (fast vs. slow). Implication of a direct pathway in the early processing of fast motion may have evolved through its behavioral relevance. information reaches the thalamus where the magnocellular layers of LGN convey most of the motion information to primary visual cortex V1 (Felleman \& Van Essen, 1991; Maunsell \& Van Essen, 1983; Van Essen \& Maunsell, 1983), which in turn projects to V5. This pathway (i.e., geniculostriate) is also thought to support awareness of visual motion through recurrent feedback to V1 (Bullier, 2001; Lamme, 2001; Pascual-Leone \& Walsh, 2001). However, this is not the only route by which motion information can reach V5. The existence of functional connections between subcortical structures such as the superior colliculus (SC) and pulvinar to V5 has been widely documented in primates (Berman \& Wurtz, 2010, 2011; Lyon, Nassi, \& Callaway, 2010; Rodman, Gross, \& Albright, 1990), and the involvement of both SC (Schneider \& Kastner, 2005) and V5 (Tootell et al., 1995; Watson et al., 1993; Zeki et al., 1991) in the processing of motion signals suggests the existence of similar connections also in humans. As opposed to the geniculostriate route, this alternative (i.e., colliculo-extrastriate) visual pathway seems to support mainly implicit visual processing in the absence of awareness. In line with this view, this pathway has been suggested to mediate the phenomenon of blindsight (Lanyon et al., 2009; Leh, Johansen-Berg, \& 
Ptito, 2006), a condition referring to the presence of residual visual abilities despite the loss of vision caused by lesions to primary visual areas (Stoerig, 2006; Weiskrantz, 1996). Indeed, blindsight patients usually report being unaware of visual stimuli presented in the scotopic area while performing above chance level when forced-choice paradigms are used (Azzopardi \& Cowey, 1998; Stoerig \& Cowey, 1997). Furthermore, this ability is particularly pronounced when moving stimuli are presented (Weiskrantz, 1986; Riddoch, 1917). Importantly, some studies revealed that blindsight patient G. Y. with intact V5 but lesioned V1 showed a greater detection and discrimination performance (also accompanied with some report of awareness) for fast moving ( $>6 \% \mathrm{sec}$ ) than slower moving stimuli. The authors interpreted these results as evidence of a recruitment of the subcortical connection bypassing V1 when fast moving stimuli, as opposed to slow moving stimuli, have to be processed. Similarly, another study showed a preserved early EEG response to fast motion, but not slow motion, when stimuli were presented in G. Y.'s blind hemifield (Ffytche, Guy, \& Zeki, 1996) corroborating the idea of a specific recruitment of direct connections to V5 only for fast motion processing. This result was however challenged by similar follow-up studies in the same patient (Benson, Guo, \& Hardiman, 1999; Holliday, Anderson, \& Harding, 1997). Conversely, stimuli moving at slow velocities may mostly rely on the activity of the geniculostriate pathway and may consequently need the integrity of V1. This is supported by the finding of a double-dissociation pattern of performance in a patient with bilateral V5 lesions but intact V1 showing higher reliability in the detection and discrimination of slow moving $\left(<6^{\circ} \mathrm{sec}\right.$ ) than fast moving stimuli (Hess, Baker, \& Zihl, 1989; Zihl, von Cramon, \& Mai, 1983). Taken together, these results suggest that the recruitment of the direct colliculo-extrastriate or the indirect geniculostriate input to V5 could strongly depend on the characteristics of motion, a phenomenon referred to as dynamic parallelism (Ffytche et al., 1996; Ffytche, Guy, \& Zeki, 1995).

Nonetheless, the existence of two segregated pathways that could be selectively activated by the presence of fast versus slow moving signals is still a matter of debate. To date, no consistent evidence for dynamic parallelism in healthy participants has been reported. For instance, although a study combining EEG and magnetoencephalography measurements revealed earlier evoked responses to fast moving stimuli over V5 as compared with V1 and a reverse temporal pattern when slow moving stimuli were presented (Ffytche et al., 1995), a subsequent magnetoencephalography study using a variety of stimulus parameters found no evidence of an early V5 response that could be explained by a direct subcortical input (Anderson, Holliday, Singh, \& Harding, 1996). Likewise, in monkeys, although intracranial recordings from area V5A revealed an earlier response latency to fast than slow moving stimuli (52 vs. 60 msec; Kawano, Shidara, Watanabe, \& Yamane, 1994), a subsequent study on V1 lesioned macaque mon- keys showed no differential latencies in area MT to slow versus fast motion (Azzopardi, Fallah, Gross, \& Rodman, 2003).

In the present TMS study, we tested the hypothesis of dynamic parallelism in two groups of healthy participants by transiently interfering with V5 or V1/V2 processing, respectively. We probed visual motion processing by applying double-pulse TMS at different SOAs while participants were asked to judge the direction of motion (2AFC) of a patch of dots moving at either $23 \% \mathrm{sec}$ (fast motion) or $4.4^{\circ} \mathrm{sec}$ (slow motion). In addition, to examine possible dissociations between objective and subjective measures of perception induced by TMS interference, participants were asked to provide a trial by trial rating of their awareness of motion. If dynamic parallelism of motion processing were effective, one would expect TMS to differently interfere with fast versus slow motion processing when $\mathrm{V} 5$ or $\mathrm{V} 1 / \mathrm{V} 2$ is being stimulated. In this case, V5-TMS interference with objective measures should occur at earlier latencies for fast than slow moving stimuli, suggesting the involvement of a more direct pathway to V5 for the processing of fast motion (i.e., colliculoextrastriate). In addition, given the crucial role played by recurrent loops through V1/V2 in visual awareness (e.g., Silvanto, Cowey, Lavie, \& Walsh, 2005), early V5TMS effects on motion processing should be more decoupled from subjective awareness for fast than slow moving stimuli, suggesting that fast motion processing relies more on implicit nonprimary visual cortex function at early stage. The reverse could occur for V1/V2-TMS, which may conversely show an earlier time window of TMS interference with slow than fast moving stimuli and greater coupling of these V1/V2-TMS effects to subjective awareness.

\section{METHODS}

\section{Participants}

Twelve individuals took part in Experiment 1 (two men; mean age $=25.1, S D=3.9)$, and 12 individuals took part in Experiment 2 (three men; mean age $=25.1, S D=$ 3.8), nine of whom participated in both experiments. Participants were selected based on their abilities to perceive moving (Experiment 1) or static (Experiment 2) TMSinduced phosphenes, which were used to identify areas V5/MT and V1/V2, respectively (see TMS and Functional Localization section for further details). All participants were naive to the purpose of the study and had normal or corrected-to-normal vision. No participant presented with contraindications to TMS (Rossi, Hallett, Rossini, Pascual-Leone, \& Safety of TMS Consensus Group, 2009), nor with a history of neurological or psychiatric disorders. Before taking part in the experiment, all participants provided written informed consent. The study was approved by the ethics committee of the College of Science and Engineering, University of Glasgow. 


\section{Experimental Design}

Each experiment consisted of three sessions conducted on three separate days (see Figure 1A). The first day served to determine TMS location and intensity per participant (see TMS and Functional Localization section) and to equate task complexity across participants and conditions for the following sessions (see Titration section). The experimental sessions were performed on

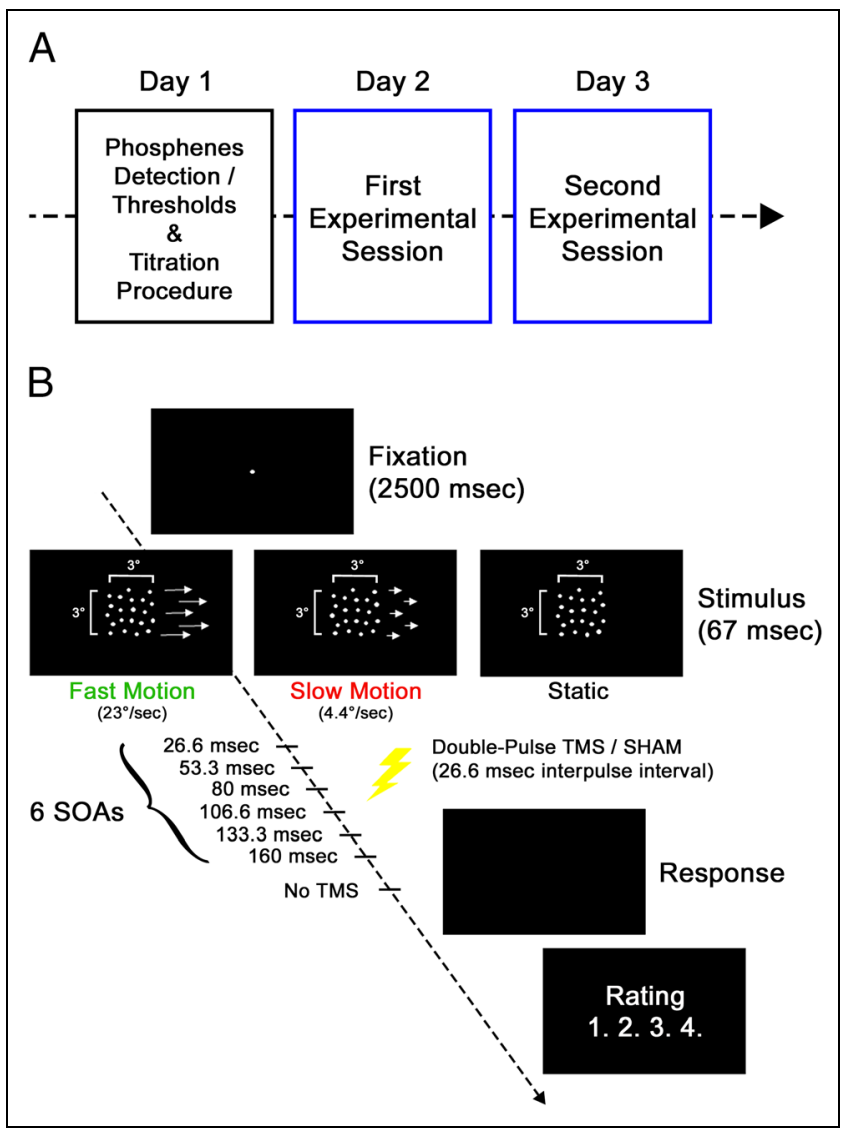

Figure 1. Experimental design and stimuli employed in two TMS experiments targeting area V5 or V1/V2, respectively (Experiments 1 and 2), while healthy participants had to discriminate direction of fast versus slow moving visual stimuli. (A) Experimental design: Each experiment consisted of three sessions. Session 1 (at Day 1) served to identify areas V5 or V1/V2 by induction of moving versus static TMS phosphenes, respectively, and to equate task complexity across conditions (fast motion and slow motion) for the following sessions. Sessions 2 and 3 served for data acquisition to examine TMS interference with fast versus slow visual motion processing (TMS intensity $<$ phosphene threshold, trials equally split across Days 2 and 3). (B) Schematic representation of a trial. Each trial began with a fixation cross $(2500 \mathrm{msec})$ followed by the presentation of a stimulus (fast motion, slow motion, or static, $67 \mathrm{msec}$, five frames). Real or SHAM double-pulse TMS was then applied at one of six possible SOAs, or alternatively, no TMS was delivered. Participants were asked to report the perceived direction of motion (by 2AFC) and to rate their awareness of motion on a 4-point scale ( $1=$ "I did not perceive any motion at all"; 2 = "I might have perceived motion but I did not have any idea of its direction"; 3 = "I did not actually see the direction of the motion, but I may bave been able to sense or guess its direction"; $4=$ "I saw the direction of the motion"). the second and third day. For those participants who took part in both Experiments 1 and 2, order of experiments was randomized (five participants starting with one experiment and four with the other).

\section{TMS and Functional Localization}

TMS was administered using a Magstim Super Rapid2 Plus1 stimulator and a figure-of-eight coil. Double-pulse TMS with an interpulse interval of $26.7 \mathrm{msec}$ was used both during the functional localization phase and during the experimental sessions.

Experiment 1 examined the effect of TMS over motion area V5. We chose to stimulate left V5 in all participants based on previous TMS studies showing more interference with motion processing after left than right hemispheric interventions (Anand, Olson, \& Hotson, 1998; Beckers \& Hömberg, 1992). Please also note that other TMS studies reported a significant and time-restricted impairment in motion detection/discrimination for stimuli presented at the center of the visual field despite unilaterally delivered V5-TMS (Koivisto, Mäntylä, \& Silvanto, 2010; Laycock, Crewther, Fitzgerald, \& Crewther, 2007; Silvanto, Lavie, \& Walsh, 2005). Furthermore, Hotson and Anand (1999) showed left V5-TMS to disrupt motion discrimination in both ipsi- and contralateral hemifields. Hence, we expected left V5-TMS to effectively interfere with processing of the chosen, centrally presented, motion stimuli (see Coherent Motion Stimuli, Task, and Apparatus section). Left V5 was localized individually at Day 1 by establishing for each participant the site over which TMS most strongly induced the perception of moving phosphenes. To this end, we first drew on an elastic cap a $6 \times 6 \mathrm{~cm}$ grid of 36 points centered $3 \mathrm{~cm}$ above and $3 \mathrm{~cm}$ to the left of the inion. Starting $3 \mathrm{~cm}$ dorsal and $4 \mathrm{~cm}$ lateral from the inion, the coil was then moved across the grid in 1-cm steps to find the site from which most reliable moving phosphenes were induced. On average, this was $3.3 \mathrm{~cm}$ above and $2.6 \mathrm{~cm}$ lateral from the inion (center of coil).

Experiment 2 examined the effects on TMS over early visual areas (V1/V2). In analogy to Experiment 1, V1/V2 was localized at Day 1 by establishing for each participant the site over which TMS induced the perception of static phosphenes covering the center of the visual field, hence optimizing interference with visual processing at the central retinotopic position where the moving stimuli were presented. To this end, we first drew on an elastic cap a $4 \times 4 \mathrm{~cm}$ grid of 16 points centered $2 \mathrm{~cm}$ above the inion. Starting from $2 \mathrm{~cm}$ above the inion, the coil was then moved across the grid in 1-cm steps to find the site from which most reliable static phosphenes were induced. On average, this was $2.6 \mathrm{~cm}$ above the inion (coil center).

For both V5 and V1/V2 localization, TMS intensity was initially set to $50 \%$ of maximum stimulator output (MSO) and then gradually increased or decreased following a 
pseudostaircase procedure based on the participants' phosphene reports. TMS was delivered at a halfway intensity between an upper boundary and a lower boundary, which were recurrently adjusted based on participants' report of phosphenes. Starting with a lower boundary of $0 \%$ MSO and an upper boundary of $100 \%$ MSO, the initial stimulation intensity was set at 50\% MSO. If participants reported the presence of a phosphene, the upper boundary was lowered to $50 \% \mathrm{MSO}$, and the stimulation intensity was consequently set at 25\% MSO. Conversely, if participants did not report the presence of a phosphene, the lower boundary was increased to $50 \% \mathrm{MSO}$, and the stimulation intensity was set at $75 \% \mathrm{MSO}$. The procedure was repeated until five reversals (i.e., five opposite responses) were obtained. The corresponding stimulation intensity was then set as the individual phosphene threshold. To confirm phosphene threshold, six additional pulses were then delivered at the chosen intensity. If participants reported a phosphene in half of the trials, the intensity was considered valid. Otherwise, it was adjusted by a factor of 1-2\% MSO, and six more pulses were delivered. The procedure was terminated when half of the pulses were associated with phosphene reports.

Nine participants perceived both moving and static phosphenes and therefore participated in both experiments. Six participants perceived either moving $(n=$ $3)$ or static phosphenes $(n=3)$ and therefore took part in Experiment 1 or Experiment 2 only. Coil orientation was individually chosen based on each participant's most consistent reports of phosphenes. This resulted in the coil handle oriented upward for both V5 and V1/V2 stimulation in the majority of participants.

During the experimental sessions, the stimulator output was set to $10 \%$ below the individual defined phosphene threshold to avoid phosphene perception. Moreover, this particular stimulation intensity was found to be effective in disrupting motion discrimination when performance accuracy at baseline was around $\sim 80 \%$ (Schwarzkopf, Silvanto, \& Rees, 2011), that is, at the difficulty level chosen for our motion stimuli (see Titration section). The average stimulator output used was 36\% MSO for V5 stimulation and $40 \% \mathrm{MSO}$ for V1/V2 stimulation.

\section{Coherent Motion Stimuli, Task, and Apparatus}

Eighty white dots (dot size: $3 \times 3$ pixels) were presented on a black background within an area of $3^{\circ} \times 3^{\circ}$ of visual angle (squared) at the center of a CRT monitor (19 in., refresh rate: $75 \mathrm{~Hz}, 1280 \times 1024$ pixel resolution). A percentage of dots could move either rightward or leftward (coherent motion) over five frames (66.7 msec) at either $23^{\circ} / \mathrm{sec}$ (fast motion) or $4.4^{\circ} / \mathrm{sec}$ (slow motion), whereas the remaining percentage of dots moved in a random manner. In addition, during the experimental sessions, static stimuli were also presented (see Figure 1B). The experiment was conducted in a dimly lit and soundcontrolled room with participants seated with their chin placed on a chinrest at a viewing distance of $57 \mathrm{~cm}$ from the monitor. A computer running E-Prime software (Version 2.0) controlled stimulus presentation and manual response collection.

\section{Titration}

In both experiments, at Day 1, a titration curve was established per motion velocity condition to individually adjust stimulus properties (i.e., motion coherence levels) to match task difficulty between fast and slow motion stimuli for the subsequent experimental sessions.

Each trial began with a fixation cross (2500 msec) followed by the presentation of a left- or rightward moving stimulus (either fast or slow motion; see Coherent Motion Stimuli, Task, and Apparatus section). After the presentation of the motion stimulus, participants were asked to indicate the perceived direction of motion (2AFC) or to guess if not perceived. Stimuli were randomly presented at 1 of 12 coherence levels (ranging from a minimum of $8 \%$ to a maximum of $62 \%$ of dots moving coherently). Participants performed three blocks of titration, each composed of 384 trials equally distributed across the two velocity conditions (fast motion, slow motion) and the 12 levels of coherence. The coherence level at which each participant performed at an accuracy of $75-85 \%$ (average over the three titration blocks) was then selected for the experimental sessions. On average, stimuli were presented at $23 \%$ of coherence for fast motion and $35 \%$ for slow motion.

\section{Experimental Session}

For each participant, slow motion and fast motion stimuli were set at the coherence rate established during titration (see Titration section). In addition, static catch stimuli (80 white dots presented centrally for $66.7 \mathrm{msec}$ within $3^{\circ} \times 3^{\circ}$ of visual angle on black background) were included in the experimental sessions (see Figure 1B).

To control for eye blinks during stimulus presentation, two electrodes were placed above and below the participants' left eye, and EOG activity was continuously recorded. Before the beginning of each experimental session, the location and the intensity of TMS was checked to confirm the validity of the parameters determined in the previous session (see TMS and Functional Localization section).

Each trial started with a fixation cross (2500 msec) followed by the presentation of a stimulus (66.7 msec; fast motion, slow motion, or static). Double-pulse TMS with an interpulse interval of $26.7 \mathrm{msec}$ was then delivered at six different delays from stimulus onset (SOAs: 26.7, 53.3, 80, 106.6, 133.3, and $160 \mathrm{msec}$ ) in either an active TMS block or a SHAM-TMS block (see Figure 1B), the latter to control for a potential influence of the TMS click on task performance. As an addition control condition, no TMS trials were also included. Participants were 
first asked to judge as fast and accurately as possible whether they perceived a rightward or a leftward motion and then to rate their perceptual awareness on the same 4-point rating scale previously used by Koivisto et al. (2010): (1) "I did not perceive any motion at all"; (2) "I might have perceived motion but I did not have any idea of its direction"; (3) "I did not actually see the direction of the motion, but I may have been able to sense or guess its direction"; (4) "I saw the direction of the motion." When a static stimulus was perceived, participants were instructed to guess in response to the first prompt and then select the first point of the perceptual awareness rating scale ("I did not perceive any motion at all").

A total of 24 stimuli was presented for each SOA (and no TMS) and for each type of stimulus (fast motion, slow motion, and static) distributed across 12 blocks. Each experimental session consisted of six blocks of TMS and six blocks of control SHAM stimulation. In SHAM blocks, stimulation was administered with the coil in the same position as in the TMS blocks but oriented perpendicular to the scalp. The order of TMS and SHAM blocks was pseudorandomized across participants within experimental sessions: Half of the blocks $(n=6)$ were administered during the first experimental session (Day 2), whereas the other half ( $n=6)$ were administered during the second experimental session (Day 3).

\section{Analysis}

Because TMS can evoke startle eye blink responses (interfering with perception), only eye blink free trials (as assessed by EOG in a poststimulus time window ranging from 0 to $100 \mathrm{msec}$ after stimulus onset) and trials with RTs $<3 S D$ from the mean were considered. Inverse efficiency scores (IES $=$ mean RTs/proportion of correct responses) were computed as a measure of objective performance, whereas the mean of rating scale scores (RSS $=$ sum of rating scores/number of trials) was used as a measure of subjective performance. These measures were analyzed per experiment using $2 \times 2 \times 6$ repeatedmeasure ANOVAs with the within-subject factors Stimulation (TMS, SHAM), Velocity (fast motion, slow motion), and TMS SOA (26.7, 53.3, 80, 106.6, 133.3, and $160 \mathrm{msec})$, followed up by simple tests where appropriate. We were a priori interested in direct comparisons of performance between TMS and SHAM trials. This resulted in a limited number of planned comparisons (i.e., six for each velocity), which were conducted using uncorrected $t$ tests. To control for Type I errors, relevant contrasts were also analyzed with Duncan's multiple range post hoc test.

Further tests included analysis of SHAM trials and static trials for control purposes. A $2 \times 6$ ANOVA with the withinsubject factors Velocity (fast motion, slow motion) and TMS SOA (six levels) was used for SHAM trials and a $2 \times 6$ ANOVA with the within-subject factors Stimulation (TMS, SHAM) and TMS SOA (six levels) was used for the analysis of static trials. No TMS trials were analyzed with a two-tailed $t$ test comparing performances between fast and slow motion. Correlation analyses (Spearman rank correlations) were performed to examine possible relationships between the effects of TMS on objective and subjective motion perception (or the absence thereof).

\section{RESULTS}

\section{Experiment 1 (V5-TMS)}

Figure 2 illustrates task performance in terms of both the objective measure (2AFC response; Figure $2 \mathrm{~A}$ ) and subjective measures (awareness of motion ratings; Figure 2B) and for both fast and slow motion stimuli (left vs. right panels) under V5-TMS (colored lines) as compared with SHAM (black line).

\section{Objective Measures}

Regarding objective performance (Figure $2 \mathrm{~A}$ ), the $2 \times 2 \times$ 6 ANOVA revealed a significant Stimulation $\times$ Velocity $\times$ TMS SOA interactions $(F(5,55)=2.967, p=.019)$, suggesting that V5-TMS had differential effects on motion processing over SOAs depending on motion velocity (left vs. right panels). We therefore conducted two separate $2 \times 6$ ANOVAs, one per motion velocity. For fast motion trials (Figure 2A, left), we found a significant Stimulation $\times$ TMS SOA interaction $(F(5,55)=2.826, p=.024)$. This was explained by significant TMS effects (TMS vs. SHAM) at the second, fourth (trend toward significance), and sixth SOA, where TMS impaired motion detection relative to SHAM (second SOA: $1003.96 \mathrm{vs} .898 .27 \mathrm{msec}, t(11)=$ $2.402, p=.035$; fourth SOA: 895.73 vs. $805.15 \mathrm{msec}, t(11)=$ $2.086, p=.061$; sixth SOA: 805.77 vs. $691.54 \mathrm{msec}, t(11)=2.739$, $p=.019)$. Duncan's multiple range post hoc test confirmed significant differences at the second and sixth SOA (second SOA: $p=.05$; fourth SOA: $p=.10$; sixth SOA: $p=.03$ ). Importantly, this effect was not driven by outliers, as TMS interference relative to SHAM was observed in the majority of participants (10 of 12 in the second SOA; see inset in Figure 2A, left, illustrating single-subject data for second SOA). The same $2 \times 6$ ANOVA on slow motion trials (Figure 2A, right) also revealed a significant Stimulation $\times$ TMS SOA interaction $(F(5,55)=2.723, p=.028)$, which however was explained by a significant TMS effect (TMS vs. SHAM) at the third SOA only, where TMS impaired performance relative to SHAM (883.85 vs. $783.54 \mathrm{msec}, t(11)=3.036$, $p=.011)$. Also in this case, Duncan's multiple range post hoc test confirmed a significant difference at the third SOA $(p=.03)$. Again, TMS interference relative to SHAM was observed in the majority of participants (10 of 12 ; see inset in Figure $2 \mathrm{~A}$, right, for individual data). In addition, the overall $2 \times 2 \times 6$ ANOVA revealed a significant main effect of TMS SOA $(F(5,55)=12.073, p<$ .001 , linear decrease of IES over time) and a significant Stimulation $\times$ TMS SOA interaction $(F(5,55)=2.522$, 

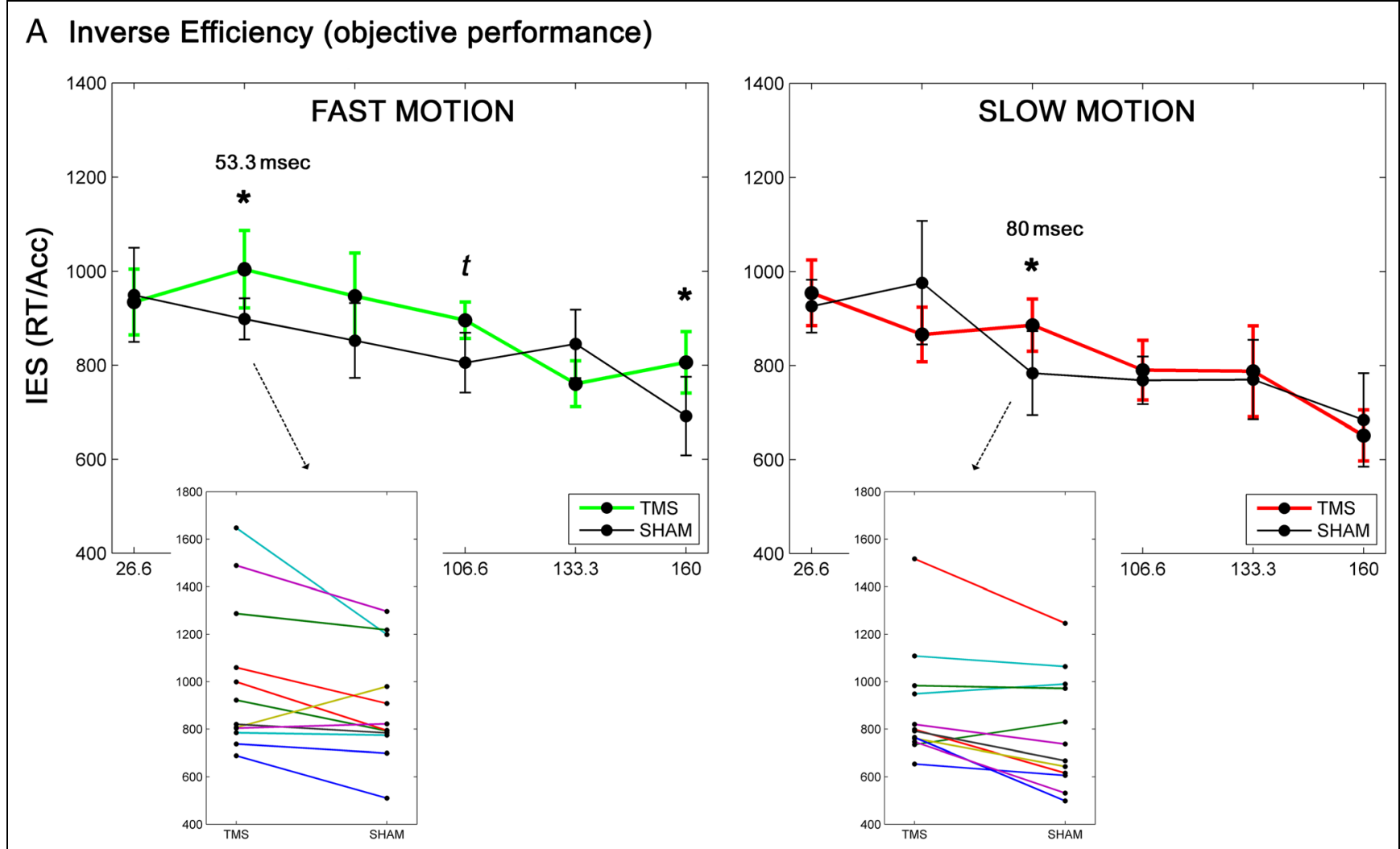

\section{B Motion Rating Scale (subjective performance)}
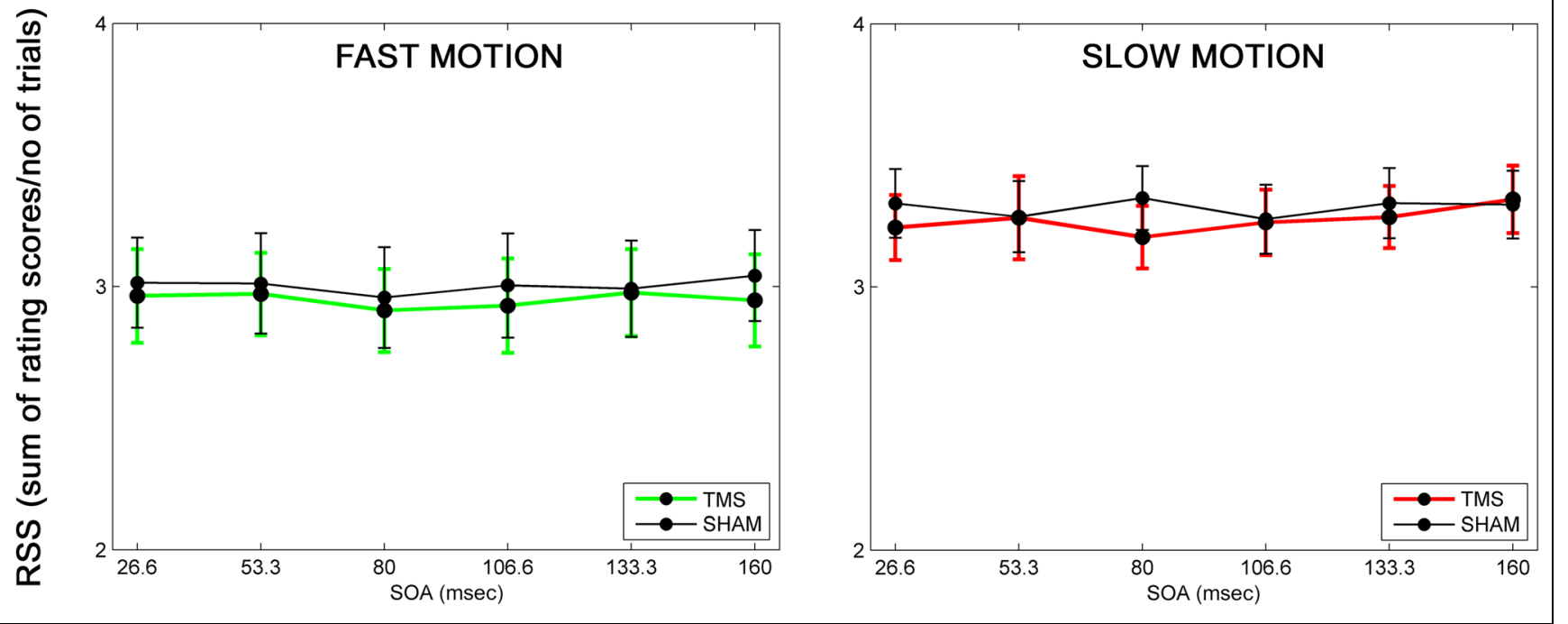

Figure 2. Interference of V5-TMS (Experiment 1) with objective and subjective motion perception. (A) Objective performance (IES) and (B) subjective performance (motion RSS) during TMS (colored lines) versus SHAM (black lines) at each SOA for fast motion (left) and slow motion (right). Error bars represent 95\% confidence interval corrected for a within-subject design (Cousineau, 2005). Note that V5-TMS interfered with fast versus slow motion at an earlier (second) versus later (third) SOA for objective performance (in A). Insets on the upper panels represent single participants' trends in TMS and SHAM blocks over the second SOA for fast motion (left inset) and third SOA for slow motion (right inset).

$p=.039)$ that was explained by a significant TMS effect over the third SOA (916.42 vs. $817.87 \mathrm{msec}, t(11)=$ $2.212, p=.049)$ when both velocities are collapsed.

To rule out that differences in performance between the two motion velocities may have been driven by differ- ences in task complexity, we also ran a $2 \times 6$ ANOVA on SHAM block trials only, with Motion velocity and TMS SOA as within-subject factors. The main effect of Velocity $(F(1,11)=0.070, p=.796)$ and the interaction Velocity $\times$ TMS SOA $(F(5,55)=0.675, p=.644)$ were both far 
from significant, whereas the main effect of TMS SOA was again significant $(F(5,55)=9.061, p<.001)$. The absence of differences between the two velocities was also evident on no TMS trials $(t(11)=-1.356, p=$ .202). Overall, this therefore confirms that participants' performances for the two types of stimuli were equated, as intended by the preexperimental titration session.

Importantly, to examine whether TMS over V5 selectively disrupted motion processing or interfered with visual processing in general, we also analyzed performance on static trials using a $2 \times 6$ ANOVA with Stimulation and TMS SOA as within-subject factors. The main effect of Stimulation, TMS SOA, and the interaction Stimulation $\times$ TMS SOA were all far from significant (all $p s>.30$ ), suggesting that TMS over V5 did selectively interfere with motion processing, without affecting visual processing in general (in contrast to V1/V2-TMS, see below).

Overall, our data indicate that V5-TMS interferes with motion processing at specific delays after motion onset depending on the velocity of motion, with fast motion being processed around $30 \mathrm{msec}$ earlier than slow motion ( $\sim 50$ vs. $80 \mathrm{msec}$ postmotion onset).

\section{Subjective Measures}

The analysis of subjective performance (Figure 2B) using the $2 \times 2 \times 6$ ANOVA with Stimulation, Velocity, and TMS SOA as within-subject factors revealed a main effect of Velocity $(F(1,11)=4.927, p=.048)$. On average, participants assigned lower confidence rating scores to perception of fast motion stimuli (mean $=2.97$ ) than to slow motion stimuli (mean $=3.27$ ). No other main effects (all $p s>.18$ ) or interactions were significant (all $p s>.40$ ).

\section{Correlations between Objective and Subjective Measures}

To investigate the relationship between objective and subjective measures, we examined correlations between SHAM-normalized measures of objective effects on perception (i.e., IES on TMS blocks minus IES on SHAM blocks; Figure 3A) and SHAM-normalized measures of subjective changes (i.e., RSS on TMS blocks minus RSS on SHAM blocks; Figure 3B) across participants on SOAs in which behavioral performances were found to be significantly affected by TMS (i.e., the second, fourth, and sixth SOA of the fast motion stimuli and the third SOA of the slow motion stimuli). The correlation results are illustrated in Figure 3C. Although for fast motion trials the TMS-induced decrease in objective measures did not correlate with TMS-induced decrease in subjective measures at any of the relevant SOAs (second SOA: Spearman $r(10)=.133, p=.681$; fourth SOA: $r(10)=$ $-.238, p=.457$; sixth SOA: $r(10)=.361, p=.249$; see Figure 3C, left, for second SOA), a significant correlation was found on the third SOA of slow motion trials (Spearman $r(10)=-.648, p=.022$; see Figure $3 \mathrm{C}$, right). In other words, although objective measures of disrupted fast motion perception by V5-TMS were decoupled from subjective measures (changes in the absence of awareness), this was not the case for slow motion for which changes in objective measures significantly correlated with changes in subjective measures, therefore indicating that the more TMS interfered with objective performance, the lower was the confidence rating.

\section{Experiment 2 (V1/V2-TMS)}

Figure 4 illustrates task performance in terms of both the objective measures (2AFC response; Figure $4 \mathrm{~A}$ ) and subjective measures (awareness of motion ratings; Figure 4B) and for both the fast and slow motion stimuli (left vs. right panels) under V1/V2-TMS (colored lines) as compared with SHAM (black line).

\section{Objective Measures}

Analysis of the objective performance measures (Figure $4 \mathrm{~A}$ ) revealed a significant main effect of Stimulation $(F(1,11)=6.342, p=.028)$. This was independent of SOA and Velocity (no interaction of Stimulation with these factors, see below), showing that V1/V2-TMS induced a general decrease of performance over all velocities and all SOAs with respect to SHAM-TMS (981.59 vs. $917.32 \mathrm{msec})$. No significant interactions were found between Stimulation $\times$ TMS SOA $(F(5,55)=0.487, p=$ $.785)$, Stimulation $\times$ Velocity $(F(1,11)=3.483, p=.09)$, and Stimulation $\times$ Velocity $\times$ TMS SOA $(F(5,55)=1.496$, $p=.206)$. Also, a significant main effect of TMS SOA was found $(F(5,55)=5.538, p<.001)$ explained by a linear decrease on IES measures from earlier to later SOAs, which was independent of stimulation (no interaction Stimulation and TMS SOA). This mirrors the TMS SOA effect found in Experiment 1 (Figure 2A) and is most likely explained by an unspecific effect of the TMS click on performance. No other main effects (all $p s>.21$ ) or interactions (all $p s>$.28) were significant.

To control for possible differences in performance between the two velocities due to differences in task complexity, a $2 \times 6$ ANOVA with Velocity and TMS SOA was again conducted on SHAM trials only. The main effect of Velocity $(F(1,11)=0.147, p=.708)$ and the interaction Velocity $\times$ TMS SOA $(F(5,55)=0.938, p=.464)$ were both not significant, whereas the main effect of TMS SOA was again significant $(F(5,55)=2.821, p=.024)$. The absence of difference between the two velocities was also evident on no TMS trials $(t(11)=-1.208, p=.252)$, confirming that performances in processing the two types of stimuli in the absence of TMS were equated as intended.

To investigate whether V1/V2-TMS had interfered with motion processing or affected visual processing in general, an additional $2 \times 6$ ANOVA with Stimulation and TMS SOA as within-subject factors was conducted on 


\section{A Inverse Efficiency (SHAM Normalized)}
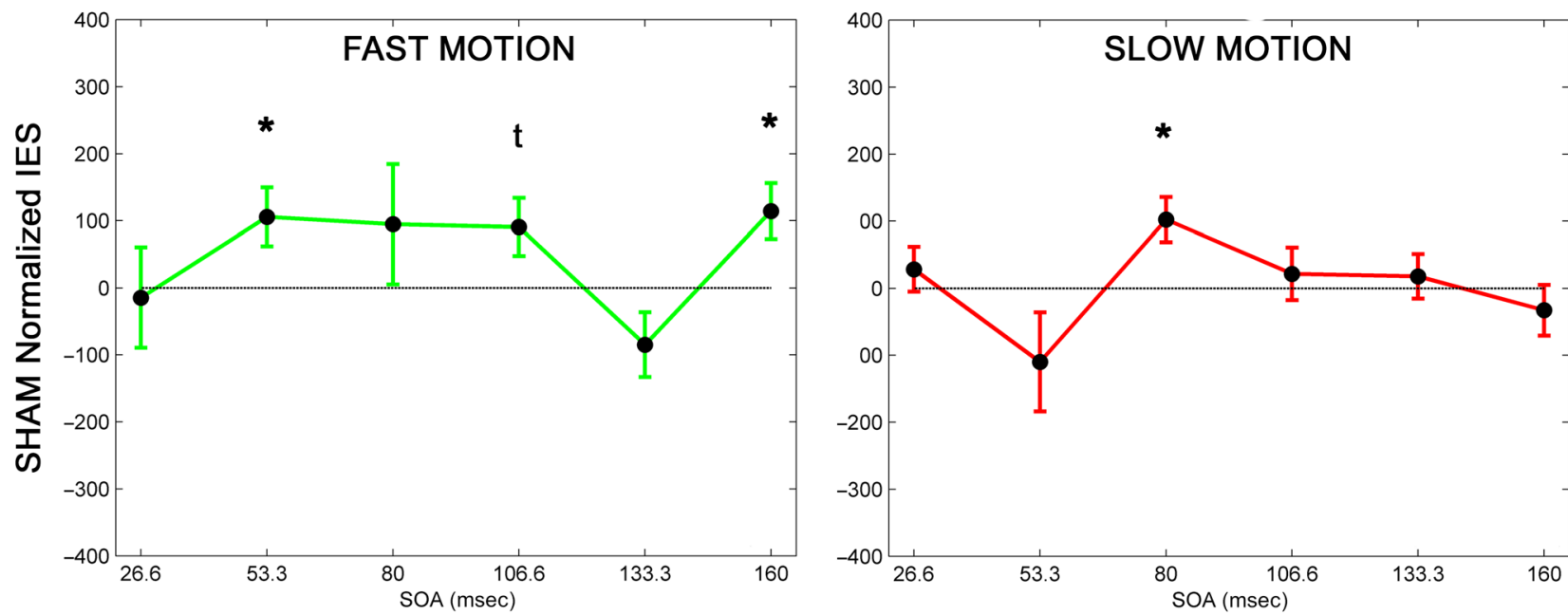

\section{B Motion Rating Scale (SHAM Normalized)}
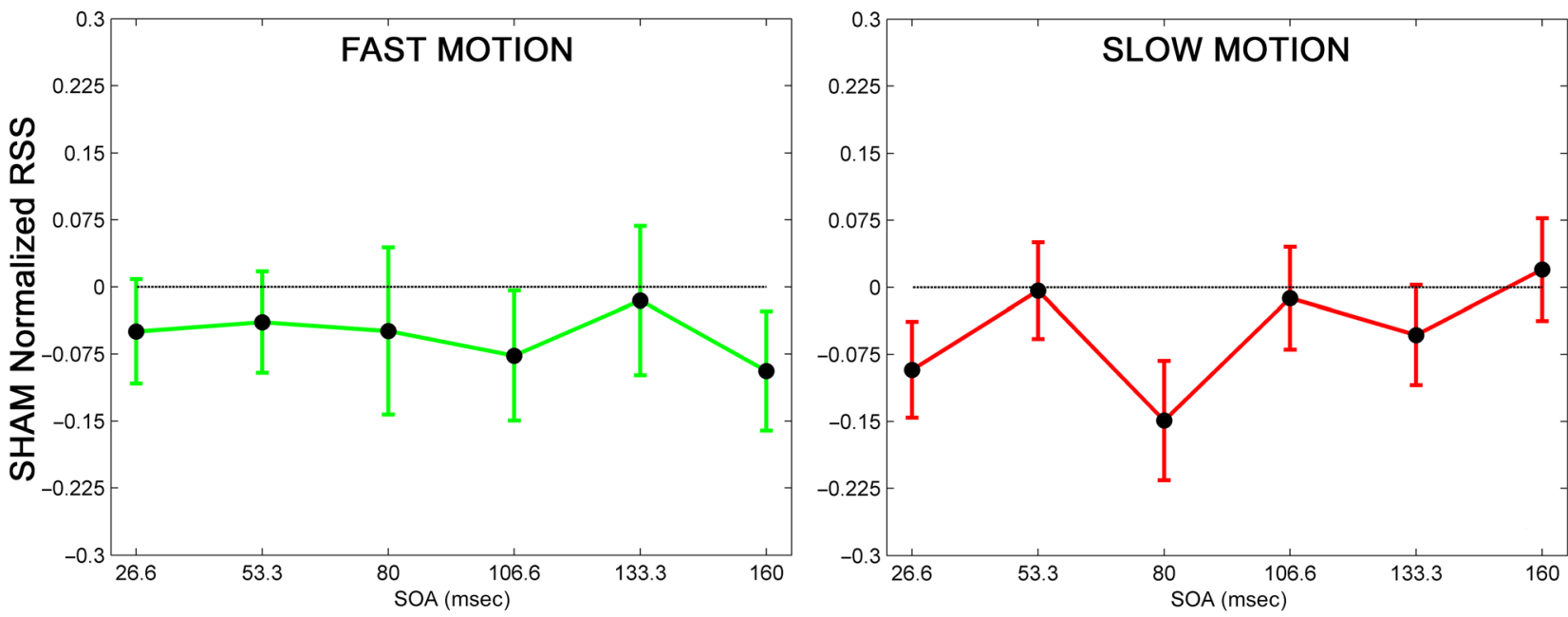

\section{A vs.B}
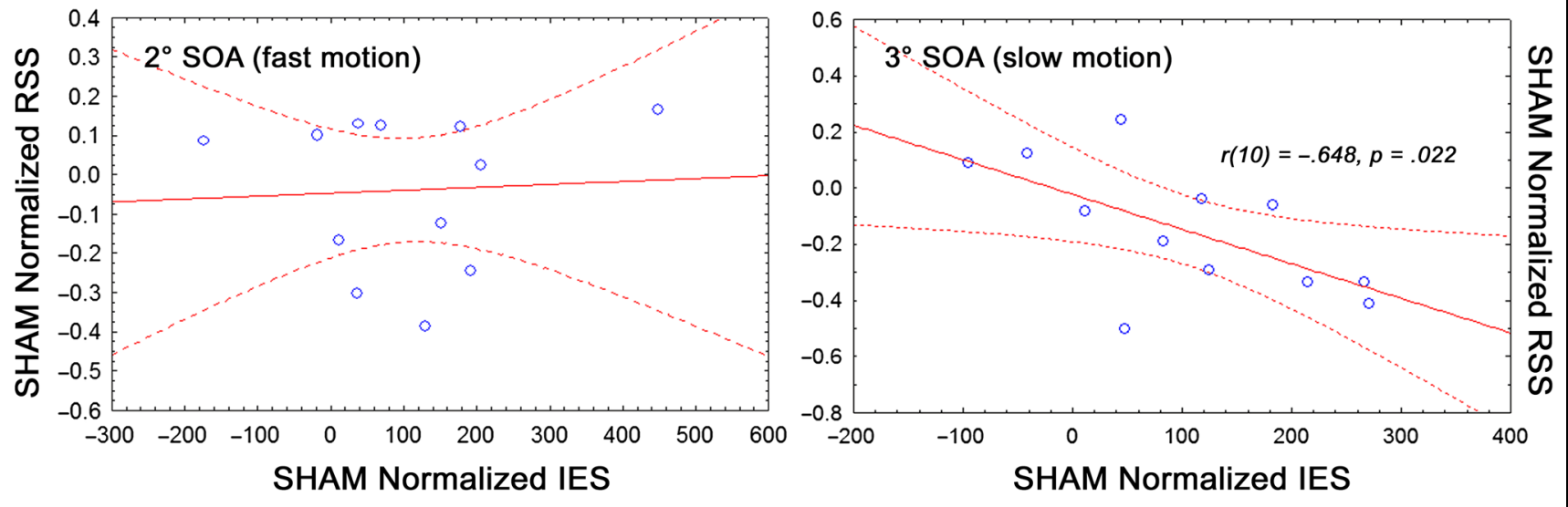

Figure 3. Relationship between interference of V5-TMS (Experiment 1) with objective versus subjective performance (SHAM-normalized).

(A) SHAM-normalized performance (i.e., TMS-SHAM) for objective and (B) subjective measures in fast motion (left) and slow motion trials (right).

(C) Correlation scatterplots (95\% confidence intervals) between SHAM-normalized objective and subjective performances (A vs. B) on the second SOA for fast motion (left inset) and the third SOA for slow motion (right inset). 

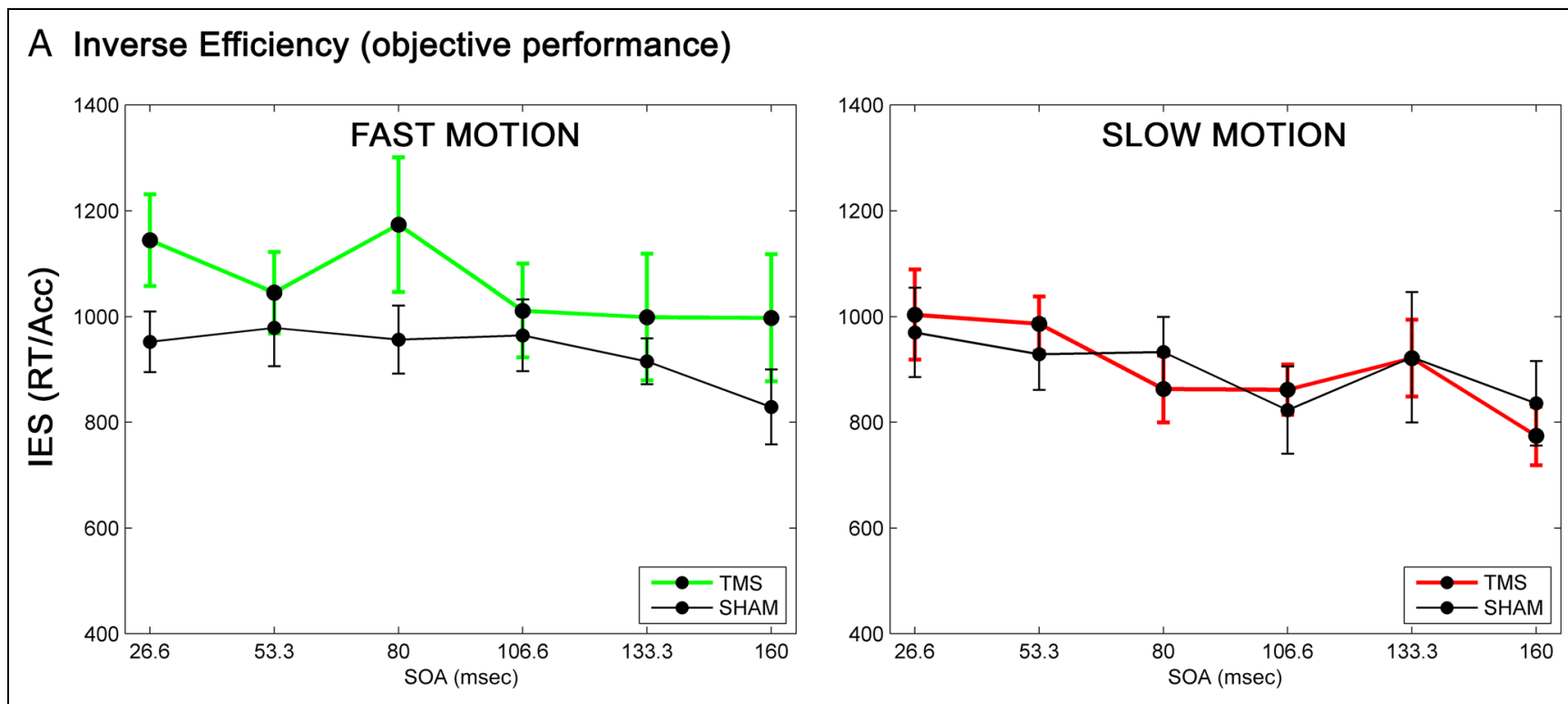

\section{B Motion Rating Scale (subjective performance)}
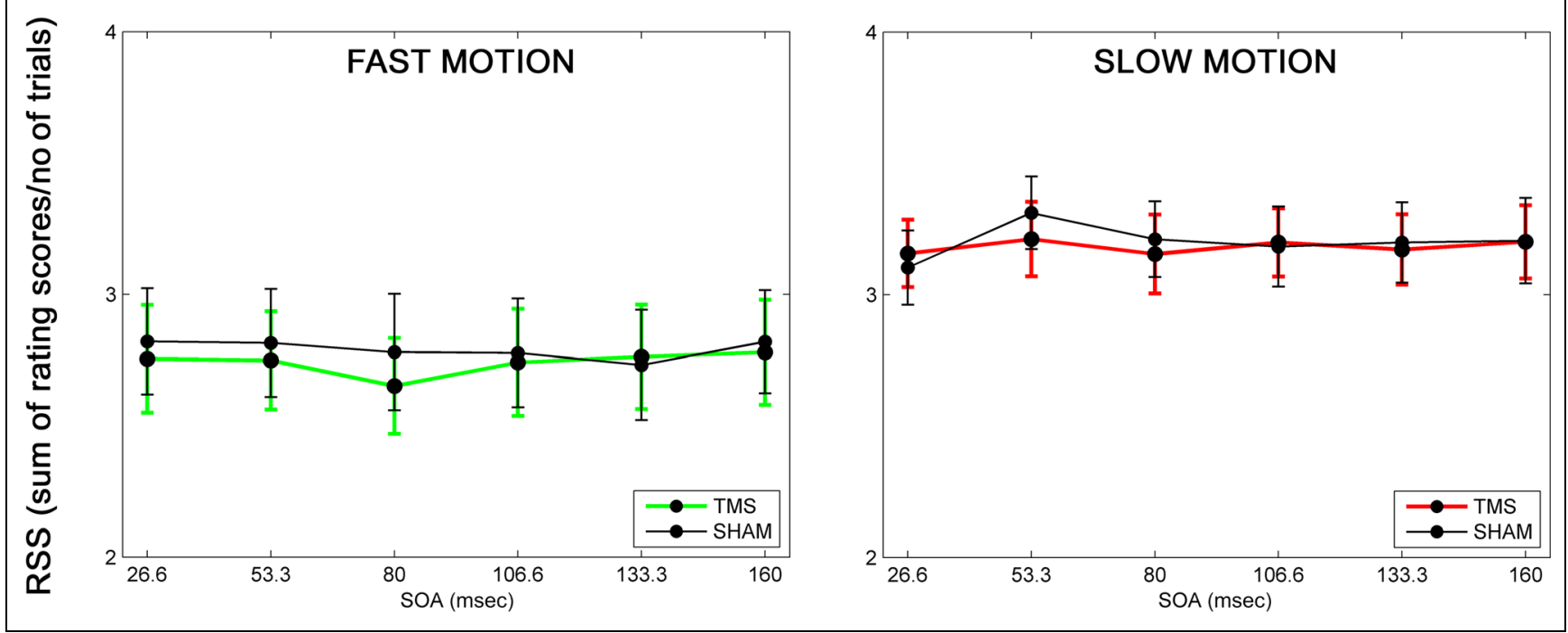

Figure 4. Interference of V1/V2-TMS (Experiment 2) with objective and subjective motion perception. (A) Objective performance (IES) and (B) subjective performance (motion RSS) during TMS (colored lines) versus SHAM (black lines) at each SOA for fast motion (left) and slow motion (right). Error bars represent 95\% confidence interval corrected for a within-subject design (Cousineau, 2005).

static trials. The main effect of Stimulation was significant $(F(1,11)=5.091, p=.045)$, suggesting that TMS over $\mathrm{V} 1 / \mathrm{V} 2$ had interfered also with the processing of static stimuli (TMS vs. SHAM: 583.48 vs. $532.89 \mathrm{msec}$ ). The main effect of TMS SOA was also significant $(F(5,55)=17.078$, $p<.001)$, explained by a linear decrease of IES measures from earlier to later SOAs, whereas the Stimulation $\times$ TMS SOA interaction was not significant $(F(5,55)=0.690$, $p=.633$ ).

Overall, this indicates that, although V5-TMS had interfered with motion processing at specific time points, V1/ V2-TMS interfered with general visual processing in a much larger time window after stimulus onset $(\sim 27-160 \mathrm{msec})$. As a consequence, inferences on the implication of V1/ V2-TMS in motion processing are limited with our data set.

\section{Subjective Measures}

The $2 \times 2 \times 6$ ANOVA with Stimulation, Velocity, and TMS SOA as within-subject factors was also conducted on RSS measures (see Figure 4B). A slight trend toward a significant main effect of Velocity $(F(1,11)=3.366$, $p=.093)$ and TMS SOA $(F(5,55)=2.211, p=.066)$ was evident. No other significant main effects (all $p s>.25$ ) or interactions (all $p s>.18$ ) were found.

\section{Correlations between Objective and Subjective Measures}

As above, correlations between SHAM-normalized measures of objective effects on perception (i.e., IES on TMS blocks minus IES on SHAM blocks; Figure 5A) and 


\section{A Inverse Efficiency (SHAM Normalized)}
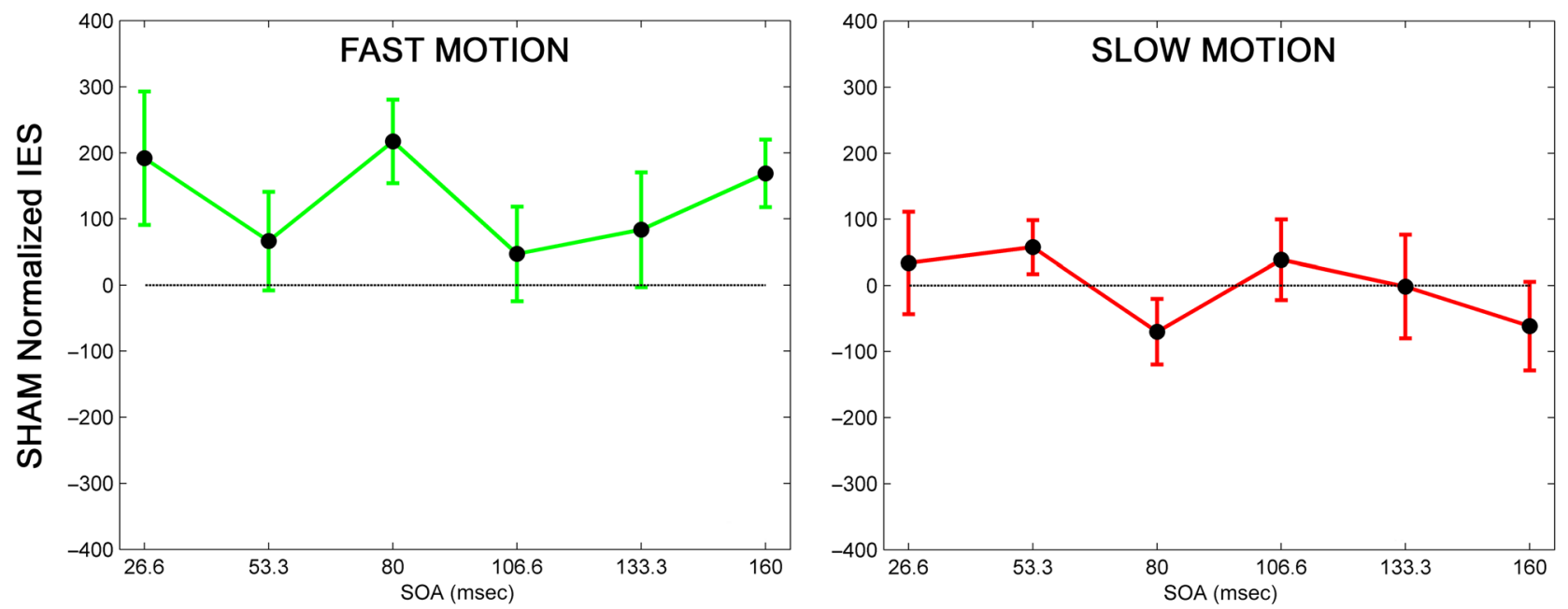

\section{B Motion Rating Scale (SHAM Normalized)}
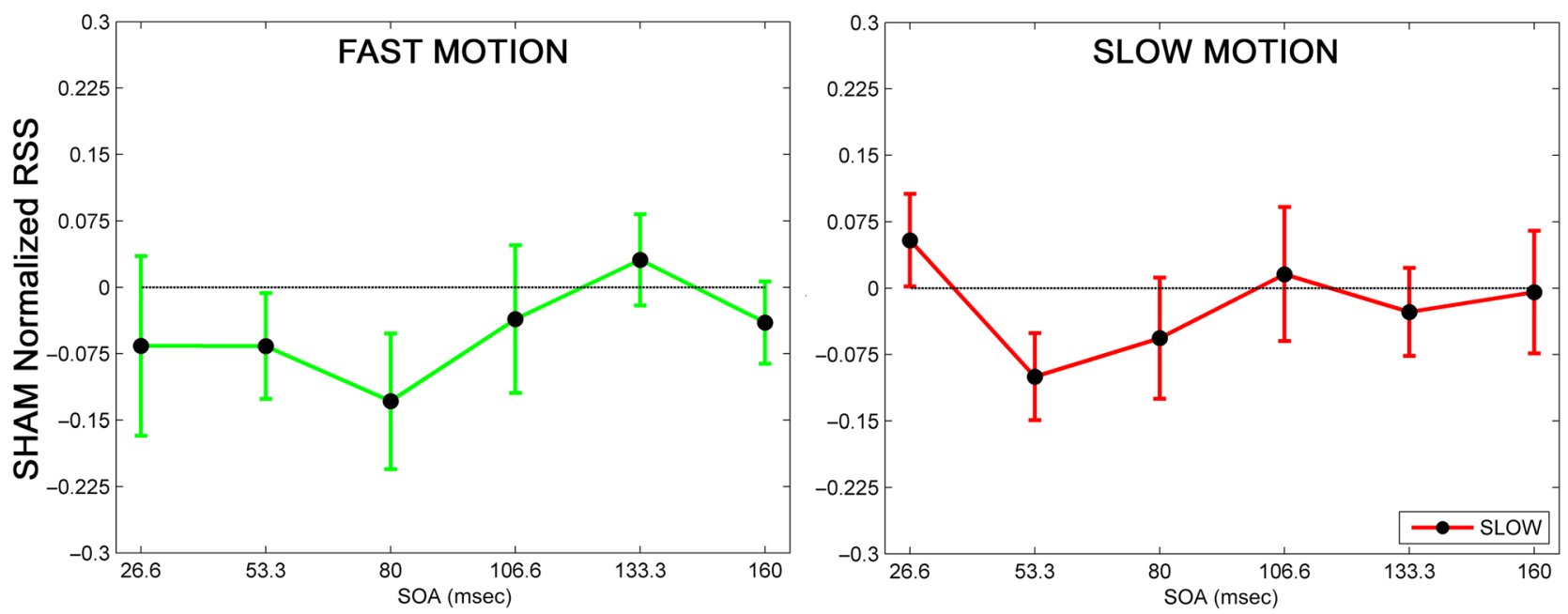

\section{Avs.B}
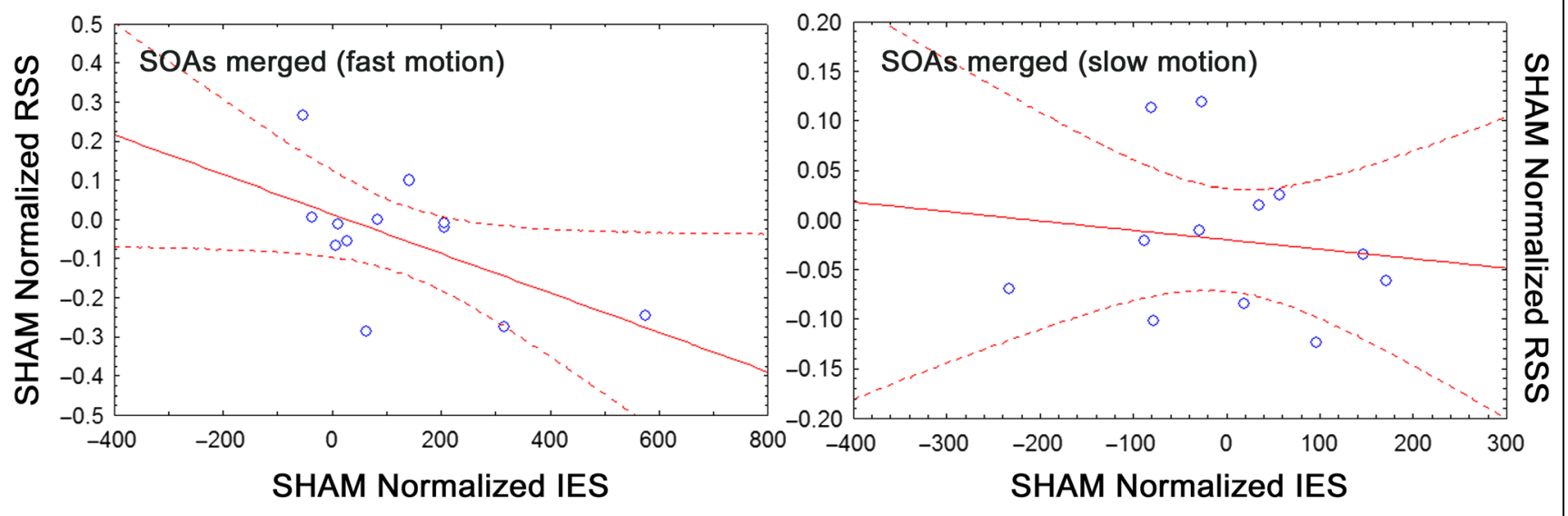

Figure 5. Relationship between interference of V1/V2-TMS (Experiment 2) with objective versus subjective performance (SHAM-normalized). (A) SHAM-normalized performance (i.e., TMS-SHAM) for objective and (B) subjective measures in fast motion (left) and slow motion trials (right). Note that V1/V2-TMS tended to interfere with motion processing across all SOAs in fast motion trials (but less so with slow motion stimuli).

(C) Correlation scatterplots (95\% confidence intervals) between SHAM-normalized objective and subjective performances (A vs. B) for fast motion (left inset) and slow motion (right inset) collapsed across SOAs. 
SHAM-normalized measures of subjective changes (i.e., RSS on TMS blocks minus RSS on SHAM blocks; Figure 5B) were examined. Because there was no effect of TMS SOA but V1/V2-TMS tended to disrupt performance over all SOAs relative to SHAM, we correlated objective and subjective measures collapsed across SOAs. The analysis did not reveal any significant correlation neither for fast motion trials (Spearman: $r(10)=-.426, p=.167$; Figure $5 \mathrm{C}$, left) nor for slow motion trials (Spearman: $r(10)=-.118, p=.713$; Figure $5 \mathrm{C}$, right). Taken together, the results of Experiment 2 indicate that with our experimental stimulation protocol, TMS applied on V1/V2 has not interfered with motion processing but rather with visual perception/processing in its global aspect.

\section{DISCUSSION}

We used TMS in a group of healthy participants to test the hypothesis of dynamic parallelism, which posits that fast parallel input to V5 bypassing V1 depends on motion characteristics (Ffytche et al., 1995, 1996). To this end, we interfered with V5 or V1/V2 processing in two separate TMS experiments (targeting V5 or V1/V2, respectively) while participants performed a motion discrimination task on fast $\left(23^{\circ} / \mathrm{sec}\right)$ or slow $\left(4.4^{\circ} / \mathrm{sec}\right)$ moving stimuli. Altogether, our results are in support of dynamic parallelism for motion processing. The results are discussed separately for each experiment below, alongside methodological considerations.

\section{Evidence for Segregated Motion Input into V5 as a Function of Motion Velocity}

Our results revealed that fast and slow moving stimuli show different timings with regard to processing by $\mathrm{V} 5$. When interfering with V5 using TMS, we found an early ( $\sim 50 \mathrm{msec})$ decrease of 2AFC performance when fast moving stimuli were processed and a later decrease ( $\sim 80 \mathrm{msec})$ with slow moving stimuli. No interference with the processing of static stimuli was found by V5TMS in any of the tested SOAs, confirming the role of V5 for the processing of motion signals (Sack et al., 2006; d'Alfonso et al., 2002; Walsh et al., 1998; Hotson et al., 1994; Beckers \& Hömberg, 1992). Interestingly, the early V5-TMS-induced decrease of 2AFC performance in the fast motion trials did not correlate with a change in motion awareness as revealed by subjective motion rating scales, whereas the later decrease in the slow motion trials was significantly correlated with a change in motion awareness.

These results suggest that fast moving stimuli could reach V5 through a more direct pathway than slow moving stimuli, given the time difference in V5-TMS interference ( $\sim 30 \mathrm{msec}$ ) found between the two velocities. Furthermore, this direct pathway seems to be independent of awareness, at least to some extent. One possibility is that fast motion information is mainly conveyed by a direct route connecting subcortical structures such as the SC to V5 and bypassing V1 (i.e., a colliculo-extrastriate route). In monkey, the existence of a functional connection between SC and V5 for motion processing has been widely documented (Berman \& Wurtz, 2010, 2011; Lyon et al., 2010; Rodman et al., 1990). In humans, a similar connection has also been suggested, given that both SC (Schneider \& Kastner, 2005) and V5 (Tootell et al., 1995; Watson et al., 1993; Zeki et al., 1991) are known to play a role in the processing of motion signals and that the exposure to a training protocol boosting SC activity specifically enhances the processing of visual motion (Grasso, Benassi, Làdavas, \& Bertini, 2016). Another possibility is that the signal bypassing V1 and directly reaching V5 could be conveyed through connections originating from the interlaminar layers of LGN, as also suggested by recent evidence on V1-lesioned patients (Ajina, Pestilli, Rokem, Kennard, \& Bridge, 2015; Schmid et al., 2010). However, the relative specialization of SC to respond to fast moving stimuli (Tohmi, Meguro, Tsukano, Hishida, \& Shibuki, 2014; Waleszczyk, Wang, Benedek, Burke, \& Dreher, 2004; Wallace, McHaffie, \& Stein, 1997) suggests that the colliculo-extrastriate route could be the pathway involved in the effects reported here.

Conversely, slow moving stimuli may reach V5 only after processing in V1 (i.e., implicating a geniculostriate route), as also suggested by the preserved ability to perceive slow but not fast motion after bilateral V5 lesions (Hess et al., 1989; Zihl et al., 1983). Our finding of a dissociation between fast and slow moving stimuli with respect to the correlation between TMS interference with objective and subjective measures of motion processing is providing further support to this notion. The fact that the early TMS interference with fast motion processing did not correlate with a change in motion awareness is indicative of a decoupling between objective and subjective analysis of fast motion signals. On the other hand, the presence of such a correlation for slow moving stimuli suggests coupling to awareness and hence supports V1 involvement with this type of motion stimulus.

Altogether, we therefore interpret our results to be in line with the idea that colliculo-extrastriate and geniculostriate pathways are selectively activated by different stimulus velocities, corroborating the view of a dynamic parallelism in the processing of motion signals (Ffytche et al., 1995, 1996). In this context, early processing of fast moving stimuli primarily relies on the activation of a direct route to $\mathrm{V} 5$, which is decoupled to some extent from their conscious experience. This suggests that this pathway is sufficient to implement implicit sensorimotor transformations necessary to quickly respond to the presence of a moving target, but not to provide a complete analysis, including awareness of it. The existence of a faster pathway specialized for the analysis of fast moving stimuli also makes sense from the perspective of an evolutionary advantage. Fast moving stimuli constitute more dangerous events and consequently require the implementation of faster motor responses 
occurring before or even in the absence of awareness. This is less important for slow moving stimuli that may reach V5 through a slower and less direct pathway, which preserves the coupling between objective and subjective experience of motion and allows for a more in-depth analysis.

Notably, the decoupling between the objective and subjective experience for fast moving stimuli resembles the decoupling between the preserved ability in detecting and discriminating fast motion reported in blindsight patients and their impaired visual awareness (Barbur, Watson, Frackowiak, \& Zeki, 1993). In this respect, it is noteworthy to consider that blindsight patient G. Y., although reporting some degree of consciousness during detection and discrimination of fast moving stimuli in Barbur et al. (1993), never reported a complete consciousness of stimuli in his blind field in several subsequent investigations (for a review, see Mazzi, Savazzi, \& Silvanto, 2017) and described his visual experience as a difficult to detail "feeling" of awareness. Therefore, even if "the hard problem of consciousness" is still strongly debated in the visual literature, it seems widely accepted that the primary visual cortex is crucial for creating conditions for awareness to arise (Mazzi et al., 2017; Silvanto, 2015; Lamme, 2001). One possibility is that a crude experience of motion could be partly achieved by nonprimary visual areas but a complete and detailed awareness would only emerge when the integrity of the geniculostriate pathway is preserved.

Interestingly, the processing of fast moving stimuli by V5-TMS was influenced not only at the second SOA (i.e., $53.3 \mathrm{msec}$ ). A decrease of performance was also evident at the third and fourth SOAs (i.e., 79.8 and $106.6 \mathrm{msec}$ ), which could reflect later input from V1 (Lamme, 2001). Finally, another decrease of performance was evident at the 160 -msec SOA in the fast motion trials. This could represent feedback signals to V5 from higher-order frontal regions such as FEFs and parietal cortex known to exert a top-down control in visual processing (Laycock et al., 2007; Ruff et al., 2006; Silvanto, Lavie, \& Walsh, 2006; Foxe \& Simpson, 2002), albeit we acknowledge the speculative nature of these interpretations. A similar decrease should also be expected for slow motion trials. However, if we consider a constant time lag between the processing of the two velocities, this should be expected in a time window beyond the SOAs considered here (i.e., $\sim 190 \mathrm{msec}$ ).

\section{No Selective Interference of V1/V2-TMS with Motion Processing}

When TMS was applied over V1/V2, we found an unspecific decrease of motion discrimination performance irrespective of stimulus velocity and SOAs. One possibility is that TMS applied over this area interfered with general visual processing, as also suggested by a significant decrease in the performance on control trials (i.e., static stimuli). Indeed, even if V1 exhibits responses to patterns of motion (McKeefry, Watson, Frackowiak, Fong, \& Zeki, 1997; Orban, Kennedy, \& Bullier, 1986), the role of this area is not strictly confined to the analysis of motion signals. It is thus reasonable to assume that TMS applied over V1/V2 could cause a broader interference with visual processing. Some previous TMS studies have shown a general disruption of visual perception over a large time window when TMS is applied to primary visual cortex (Kammer, Puls, Strasburger, Hill, \& Wichmann, 2005; Amassian et al., 1998; Amassian et al., 1989). Others reported a more restricted window of interference with motion processing even when TMS was applied over V1 (Laycock et al., 2007; Silvanto, Lavie, et al., 2005; Beckers \& Zeki, 1995; Beckers \& Hömberg, 1992) with however a large variability with regard to the timing of this window across studies. More specifically, some of these studies reported early interference at $\sim 60-70 \mathrm{msec}$ (Beckers \& Zeki, 1995; Beckers \& Hömberg, 1992), whereas others found a later window of interference at around $\sim 100$ 120 msec poststimulus onset (Silvanto, Lavie, et al., 2005; Hotson et al., 1994). One explanation for the discrepancy between our findings and previous findings with regard to the time window of V1 interference are differences in the TMS protocol used. We used a doublepulse stimulation protocol with a relative large interpulse interval (i.e., $26.7 \mathrm{msec}$ ) that could have had a much stronger interference effect when applied over V1. In accordance with this, a previous TMS study using a similar protocol (Koivisto et al., 2010) also showed a temporally unspecific disruption of motion processing over the majority of tested time intervals when TMS was applied over V1 and a much more temporally selective disruption for TMS over V5.

\section{Methodological Considerations}

The main aim of this study was to directly compare the involvement of V5 and V1/V2 in the processing of different motion velocities (fast vs. slow) using TMS. Previous studies with similar aims and using similar methodologies employed a variety of different motion velocities, but none of these studies directly contrasted the effects of TMS on motion performance at fast and slow velocities. Given the variety of results obtained across the different studies, it is somewhat difficult to identify the origin of these differences in results, that is, whether due to the various velocities used or being the result of other important methodological discrepancies between studies. For instance, Koivisto et al. (2010) used a very low stimulus velocity $\left(\sim 1^{\circ} / \mathrm{sec}\right)$ and did not find any early influence of TMS on V5. A similar result was reported by Silvanto, Lavie, et al. (2005), with stimuli moving at $\sim 2 \%$ sec. Conversely, Beckers and Zeki (1995), using dots moving at $11.5^{\circ} / \mathrm{sec}$, revealed an early time window of interference over V5 ( $20 \mathrm{msec})$ that preceded the one found for 
V1. Also Beckers and Hömberg (1992) and Laycock et al. (2007) reported an early V5-TMS induced disruption of motion discrimination but using lower stimulus velocities (i.e., $4.5^{\circ} / \mathrm{sec}$ and $1.75^{\circ} / \mathrm{sec}$, respectively). However, these results were not confirmed by other studies using dots moving at either $9^{\circ} / \mathrm{sec}$ (Sack et al., 2006) or $30^{\circ} / \mathrm{sec}$ (Hotson \& Anand, 1999) and reporting instead V5-TMS interference with motion processing at very late poststimulus time windows ( $>100 \mathrm{msec}$ ) only.

Here, building on these studies, we show that V5 differentially processes slow and fast motion by using a set of control conditions, which we consider important for enabling to discard possible confounds (and that were not always implemented in previous TMS studies on the same topic). First, we compared the results obtained by TMS with a control SHAM condition that enabled to rule out the influence of the auditory click associated with TMS on the participants' performance. Second, the use of concurrent EOG recordings enabled us to exclude TMS-induced eye blinks as the origin of the performance decrease in the presence of a TMS pulse (Jacobs, de Graaf, \& Sack, 2014). Finally, the inclusion of static stimuli (catch or control trials) allowed to pinpoint interference with motion processing per se, as opposed to visual processing in its global aspect.

\section{Conclusions}

Our results support the idea that fast and slow moving stimuli are not processed by the same pathways, corroborating that parallel processing of motion signals through segregated routes is dynamically deployed, depending on motion characteristics (dynamic parallelism). The latency difference in V5 processing between fast and slow moving stimuli ( $\sim 50 \mathrm{msec}$ vs. $80 \mathrm{msec}$ ) suggest an extra relay for slow motion stimuli, likely involving V1 (given the decoupling of objective from subjective changes to motion processing for fast but not slow moving stimuli). This is in line with the idea that motion signals can reach V5 through independent and segregated pathways.

\section{Acknowledgments}

This research received no specific grant from any funding agency in the public, commercial, or not-for-profit sectors.

Reprint requests should be sent to Paolo A. Grasso, CIMeC, Centre for Mind/Brain Science, University of Trento, Via Delle Regole 101, Mattarello, Trentino, Italy, or via e-mail: paolo.grasso@ unitn.it or Stephanie Morand, School of Life Sciences, College of Medical, Veterinary and Life Sciences, University of Glasgow, University Avenue, Glasgow, United Kingdom, or via e-mail: stephanie. morand@glasgow.ac.uk.

\section{REFERENCES}

Ajina, S., Pestilli, F., Rokem, A., Kennard, C., \& Bridge, H. (2015). Human blindsight is mediated by an intact geniculoextrastriate pathway. elife, 4, e08935.
Amassian, V. E., Cracco, R. Q., Maccabee, P. J., Cracco, J. B. Rudell, A., \& Eberle, L. (1989). Suppression of visual perception by magnetic coil stimulation of human occipital cortex. Electroencephalography and Clinical Neurophysiology/Evoked Potentials, 74, 458-462.

Amassian, V. E., Cracco, R. Q., Maccabee, P. J., Cracco, J. B., Rudell, A. P., \& Eberle, L. (1998). Transcranial magnetic stimulation in study of the visual pathway. Journal of Clinical Neurophysiology, 15, 288-304.

Anand, S., Olson, J. D., \& Hotson, J. R. (1998). Tracing the timing of human analysis of motion and chromatic signals from occipital to temporo-parieto-occipital cortex: A transcranial magnetic stimulation study. Vision Research, 38, 2619-2627.

Anderson, S. J., Holliday, I. E., Singh, K. D., \& Harding, G. F. (1996). Localization and functional analysis of human cortical area V5 using magneto-encephalography. Proceedings of the Royal Society B: Biological Sciences, 263, 423-431.

Azzopardi, P., \& Cowey, A. (1998). Blindsight and visual awareness. Consciousness and Cognition, 7, 292-311.

Azzopardi, P., Fallah, M., Gross, C. G., \& Rodman, H. R. (2003). Response latencies of neurons in visual areas MT and MST of monkeys with striate cortex lesions. Neuropsychologia, 41, 1738-1756.

Barbur, J. L., Watson, J. D., Frackowiak, R. S., \& Zeki, S. (1993). Conscious visual perception without V1. Brain, 116, 1293-1302.

Beckers, G., \& Hömberg, V. (1992). Cerebral visual motion blindness: Transitory akinetopsia induced by transcranial magnetic stimulation of human area V5. Proceedings of the Royal Society B: Biological Sciences, 249, 173-178.

Beckers, G., \& Zeki, S. (1995). The consequences of inactivating areas V1 and V5 on visual motion perception. Brain, 118, 49-60.

Benson, P. J., Guo, K., \& Hardiman, M. J. (1999). Cortical evoked potentials due to motion contrast in the blind hemifield. NeuroReport, 10, 3595-3600.

Berman, R. A., \& Wurtz, R. H. (2010). Functional identification of a pulvinar path from superior colliculus to cortical area MT. Journal of Neuroscience, 30, 6342-6354.

Berman, R. A., \& Wurtz, R. H. (2011). Signals conveyed in the pulvinar pathway from superior colliculus to cortical area MT. Journal of Neuroscience, 31, 373-384.

Bullier, J. (2001). Feedback connections and conscious vision. Trends in Cognitive Sciences, 5, 369-370.

Cousineau, D. (2005). Confidence intervals in within-subject designs: A simpler solution to Loftus and Masson's method. Tutorials in Quantitative Methods for Psychology, 1, 42-45.

d'Alfonso, A. A., van Honk, J., Schutter, D. J., Caffe, A. R., Postma, A., \& de Haan, E. H. (2002). Spatial and temporal characteristics of visual motion perception involving $\mathrm{V} 5$ visual cortex. Neurological Research, 24, 266-270.

Felleman, D. J., \& Van Essen, D. C. (1991). Distributed hierarchical processing in the primate cerebral cortex. Cerebral Cortex, 1, 1-47.

Ffytche, D. H., Guy, C. N., \& Zeki, S. (1995). The parallel visual motion inputs into areas v1 and v 5 of human cerebral cortex. Brain, 118, 1375-1394.

Ffytche, D. H., Guy, C. N., \& Zeki, S. (1996). Motion specific responses from a blind hemifield. Brain, 119, 1971-1982.

Foxe, J. J., \& Simpson, G. V. (2002). Flow of activation from V1 to frontal cortex in humans: A framework for defining "early" visual processing. Experimental Brain Research, 142, 139-150.

Grasso, P. A., Benassi, M., Làdavas, E., \& Bertini, C. (2016). Audio-visual multisensory training enhances visual processing of motion stimuli in healthy participants: An 
electrophysiological study. European Journal of Neuroscience, 44, 2748-2758.

Hess, R. H., Baker, C. L., \& Zihl, J. (1989). The "motion-blind" patient: Low-level spatial and temporal filters. Journal of Neuroscience, 9, 1628-1640.

Holliday, I. E., Anderson, S. J., \& Harding, G. F. A. (1997). Magnetoencephalographic evidence for non-geniculostriate visual input to human cortical area V5. Neuropsychologia, 35, 1139-1146.

Hotson, J. R., \& Anand, S. (1999). The selectivity and timing of motion processing in human temporo-parieto-occipital and occipital cortex: A transcranial magnetic stimulation study. Neuropsychologia, 37, 169-179.

Hotson, J. R., Braun, D., Herzberg, W., \& Boman, D. (1994). Transcranial magnetic stimulation of extrastriate cortex degrades human motion direction discrimination. Vision Research, 34, 2115-2123.

Jacobs, C., de Graaf, T. A., \& Sack, A. T. (2014). Two distinct neural mechanisms in early visual cortex determine subsequent visual processing. Cortex, 59, 1-11.

Kammer, T., Puls, K., Strasburger, H., Hill, N. J., \& Wichmann, F. A. (2005). Transcranial magnetic stimulation in the visual system. I. The psychophysics of visual suppression. Experimental Brain Research, 160, 118-128.

Kawano, K., Shidara, M., Watanabe, Y., \& Yamane, S. (1994). Neural activity in cortical area MST of alert monkey during ocular following responses. Journal of Neurophysiology, 71, 2305-2324.

Koivisto, M., Mäntylä, T., \& Silvanto, J. (2010). The role of early visual cortex (V1/V2) in conscious and unconscious visual perception. Neuroimage, 51, 828-834.

Lamme, V. A. F. (2001). Blindsight: The role of feedforward and feedback corticocortical connections. Acta Psychologica, 107, 209-228.

Lanyon, L. J., Giaschi, D., Young, S. A., Fitzpatrick, K., Diao, L., Bjornson, B. H., et al. (2009). Combined functional MRI and diffusion tensor imaging analysis of visual motion pathways. Journal of Neuro-Ophthalmology, 29, 96-103.

Laycock, R., Crewther, D. P., Fitzgerald, P. B., \& Crewther, S. G. (2007). Evidence for fast signals and later processing in human V1/V2 and V5/MT+: A TMS study of motion perception. Journal of Neurophysiology, 98, 1253-1262.

Leh, S. E., Johansen-Berg, H., \& Ptito, A. (2006). Unconscious vision: New insights into the neuronal correlate of blindsight using diffusion tractography. Brain, 129, 1822-1832.

Lyon, D. C., Nassi, J. J., \& Callaway, E. M. (2010). A disynaptic relay from superior colliculus to dorsal stream visual cortex in macaque monkey. Neuron, 65, 270-279.

Maunsell, J. H., \& Van Essen, D. C. (1983). Functional properties of neurons in middle temporal visual area of the macaque monkey. I. Selectivity for stimulus direction, speed, and orientation. Journal of Neurophysiology, 49, 1127-1147.

Mazzi, C., Savazzi, S., \& Silvanto, J. (2017). On the "blindness" of blindsight: What is the evidence for phenomenal awareness in the absence of primary visual cortex (V1)? Neuropsychologia. doi:10.1016/j.neuropsychologia.2017.10.029.

McKeefry, D. J., Watson, J. D., Frackowiak, R. S., Fong, K., \& Zeki, S. (1997). The activity in human areas V1/V2, V3, and V5 during the perception of coherent and incoherent motion. Neuroimage, 5, 1-12.

Newsome, W. T., \& Pare, E. B. (1988). A selective impairment of motion perception following lesions of the middle temporal visual area (MT). Journal of Neuroscience, 8, 2201-2211.

Newsome, W. T., Wurtz, R. H., Dürsteler, M. R., \& Mikami, A. (1985). Deficits in visual motion processing following ibotenic acid lesions of the middle temporal visual area of the macaque monkey. Journal of Neuroscience, 5, 825-840.
Orban, G. A., Kennedy, H., \& Bullier, J. (1986). Velocity sensitivity and direction selectivity of neurons in areas V1 and V2 of the monkey: Influence of eccentricity. Journal of Neurophysiology, 56, 462-480.

Pascual-Leone, A., \& Walsh, V. (2001). Fast backprojections from the motion to the primary visual area necessary for visual awareness. Science, 292, 510-512.

Riddoch, G. (1917). Dissociation of visual perceptions due to occipital injuries, with especial reference to appreciation of movement. Brain, 40, 15-57.

Rodman, H. R., Gross, C. G., \& Albright, T. D. (1990). Afferent basis of visual response properties in area MT of the macaque. II. Effects of superior colliculus removal. Journal of Neuroscience, 10, 1154-1164.

Rossi, S., Hallett, M., Rossini, P. M., Pascual-Leone, A., \& Safety of TMS Consensus Group. (2009). Safety, ethical considerations, and application guidelines for the use of transcranial magnetic stimulation in clinical practice and research. Clinical Neurophysiology, 120, 2008-2039.

Ruff, C. C., Blankenburg, F., Bjoertomt, O., Bestmann, S., Freeman, E., Haynes, J. D., et al. (2006). Concurrent TMS-fMRI and psychophysics reveal frontal influences on human retinotopic visual cortex. Current Biology, 16, 1479-1488.

Sack, A. T., Kohler, A., Linden, D. E. J., Goebel, R., \& Muckli, L. (2006). The temporal characteristics of motion processing in hMT/V5+: Combining fMRI and neuronavigated TMS. Neuroimage, 29, 1326-1335.

Schmid, M. C., Mrowka, S. W., Turchi, J., Saunders, R. C., Wilke, M., Peters, A. J., et al. (2010). Blindsight depends on the lateral geniculate nucleus. Nature, 466, 373-377.

Schneider, K. A., \& Kastner, S. (2005). Visual responses of the human superior colliculus: A high-resolution functional magnetic resonance imaging study. Journal of Neurophysiology, 94, 2491-2503.

Schwarzkopf, D. S., Silvanto, J., \& Rees, G. (2011). Stochastic resonance effects reveal the neural mechanisms of transcranial magnetic stimulation. Journal of Neuroscience, $31,3143-3147$.

Silvanto, J. (2015). Why is "blindsight" blind? A new perspective on primary visual cortex, recurrent activity and visual awareness. Consciousness and Cognition, 32, 15-32.

Silvanto, J., Cowey, A., Lavie, N., \& Walsh, V. (2005). Striate cortex (V1) activity gates awareness of motion. Nature Neuroscience, 8, 143-144.

Silvanto, J., Lavie, N., \& Walsh, V. (2005). Double dissociation of V1 and V5/MT activity in visual awareness. Cerebral Cortex, $15,1736-1741$.

Silvanto, J., Lavie, N., \& Walsh, V. (2006). Stimulation of the human frontal eye fields modulates sensitivity of extrastriate visual cortex. Journal of Neurophysiology, 96, 941-945.

Stoerig, P. (2006). Blindsight, conscious vision, and the role of primary visual cortex. Progress in Brain Research, 155 217-234

Stoerig, P., \& Cowey, A. (1997). Blindsight in man and monkey. Brain, 120, 535-559.

Tohmi, M., Meguro, R., Tsukano, H., Hishida, R., \& Shibuki, K. (2014). The extrageniculate visual pathway generates distinct response properties in the higher visual areas of mice. Current Biology, 24, 587-597.

Tootell, R. B., Reppas, J. B., Kwong, K. K., Malach, R., Born, R. T., Brady, T. J., et al. (1995). Functional analysis of human MT and related visual cortical areas using magnetic resonance imaging. Journal of Neuroscience, 15, 3215-3230.

Van Essen, D. C., \& Maunsell, J. H. R. (1983). Hierarchical organization and functional streams in the visual cortex. Trends in Neurosciences, 6, 370-375.

Waleszczyk, W. J., Wang, C., Benedek, G., Burke, W., \& Dreher, B. (2004). Motion sensitivity in cat's superior colliculus: Contribution 
of different visual processing channels to response properties of collicular neurons. Acta Neurobiologiae Experimentalis, 64, 209-228.

Wallace, M. T., McHaffie, J. G., \& Stein, B. E. (1997). Visual response properties and visuotopic representation in the newborn monkey superior colliculus. Journal of Neurophysiology, 78, 2732-2741.

Walsh, V., Ellison, A., Battelli, L., \& Cowey, A. (1998). Task-specific impairments and enhancements induced by magnetic stimulation of human visual area V5. Proceedings of the Royal Society B: Biological Sciences, 265, 537-543.

Watson, J. D. G., Myers, R., Frackowiak, R. S. J., Hajnal, J. V., Woods, R. P., Mazziotta, J. C., et al. (1993). Area V5 of the human brain: Evidence from a combined study using positron emission tomography and magnetic resonance imaging. Cerebral Cortex, 3, 79-94.

Weiskrantz, L. (1986). Blindsight: A case study and implications. New York: Oxford University Press.

Weiskrantz, L. (1996). Blindsight revisited. Current Opinion in Neurobiology, 6, 215-220.

Zeki, S., Watson, J. D., Lueck, C. J., Friston, K. J., Kennard, C., \& Frackowiak, R. S. (1991). A direct demonstration of functional specialization in human visual cortex. Journal of Neuroscience, 11, 641-649.

Zihl, J., von Cramon, D., \& Mai, N. (1983). Selective disturbance of movement vision after bilateral brain damage. Brain, 106, 313-340. 